\title{
A note on the degree of approximation of functions belonging to certain Lipschitz class by almost Riesz means
}

\author{
Uaday Singh and Arti Rathore
}

\begin{abstract}
The problem of obtaining degree of approximation for the $2 \pi$-periodic functions in the weighted Lipschitz class $W\left(L^{p}, \xi(t)\right)(p \geq 1)$ by Riesz means of the Fourier series have been studied by various investigators under $L^{p}$-norm. Recently, Deepmala and Piscoran [Approximation of signals(functions) belonging to certain Lipschitz classes by almost Riesz means of its Fourier series, J. Inequal. Appl., (2016), 2016:163. DOI 10.1186/s13660-016-1101-5] obtained a result on degree of approximation for weighted Lipschitz class by Riesz means. In this note, we extend this study to the weighted $L^{p}$-norm which in turn improves some of the previous results. We also derive some corollaries from our result.
\end{abstract}

Mathematics Subject Classification (2010): 42A10, 42A24, 41A25.

Keywords: Fourier series, degree of approximation, weighted $L^{p}$-norm, generalized Minkowski inequality, almost Riesz means.

\section{Introduction}

Let $f$ be a $2 \pi$-periodic function belonging to the space $L^{p}:=L^{p}[0,2 \pi](p \geq 1)$. Then the trigonometric Fourier series of $f$ is defined as

$$
f(x) \sim \frac{a_{0}}{2}+\sum_{k=1}^{\infty}\left(a_{k} \cos k x+b_{k} \sin k x\right)
$$

and the $n^{\text {th }}$ partial sum of the Fourier series of $f$, given by

$$
s_{n}(f ; x)=\frac{a_{0}}{2}+\sum_{k=1}^{n}\left(a_{k} \cos k x+b_{k} \sin k x\right), n \in N \text { with } s_{0}(f ; x)=a_{0} / 2,
$$


is called the trigonometric polynomial of degree or order $n$ (see [11]). Throughout this paper $\|.\|_{p}$ will denote the $L^{p}$ norm defined by

$$
\|f\|_{p}=\left\{\begin{array}{cl}
\left(\frac{1}{2 \pi} \int_{0}^{2 \pi}|f(x)|^{p} d x\right)^{\frac{1}{p}}, & p \geq 1 \\
e s s \sup _{0 \leq x \leq 2 \pi}|f(x)|, & p=\infty .
\end{array}\right.
$$

The following subclasses of $L^{p}[0,2 \pi]$ - space are well known in the literature.

A function $f \in \operatorname{Lip} \alpha$, if

$$
f(x+t)-f(x)=O\left(t^{\alpha}\right), \text { for } 0<\alpha \leq 1, t>0 .
$$

A function $f \in \operatorname{Lip}(\alpha, p)$, if

$$
\|f(x+t)-f(x)\|_{p}=O\left(t^{\alpha}\right), \text { for } 0<\alpha \leq 1, p \geq 1, t>0 .
$$

Given a positive increasing function $\xi(t)$, a function $f \in \operatorname{Lip}(\xi(t), p)$, if

$$
\|f(x+t)-f(x)\|_{p}=O(\xi(t)), \text { for } p \geq 1, t>0,
$$

and $f \in W\left(L^{p}, \xi(t)\right)$, if

$$
\left\|(f(x+t)-f(x)) \sin ^{\beta}\left(\frac{x}{2}\right)\right\|_{p}=O(\xi(t)), \text { for } \beta \geq 0, p \geq 1, t>0 .
$$

We have the following inclusions:

$$
\operatorname{Lip} \alpha \subseteq \operatorname{Lip}(\alpha, p) \subseteq \operatorname{Lip}(\xi(t), p) \subseteq W\left(L^{p}, \xi(t)\right)
$$

for all $0<\alpha \leq 1$ and $p \geq 1$. Khan [2] was the first to use the weight function of the form $\sin ^{\beta p}(x / 2)$.

We obtain the degree of approximation of a function $f \in L^{p}$-space by a trigonometric polynomial $\tau_{n}(f ; x)$ of degree $n$ in $L^{p}$-norm by measuring the deviation $\left\|\tau_{n}(f ; x)-f(x)\right\|_{p}$. This method of approximation is called the trigonometric Fourier approximation. The $\tau_{n}(f ; x)$ is called Fourier approximant of $f$.

A bounded sequence $\left\{s_{n}\right\}$ is said to be almost convergent to a limit $s$, if

$$
\lim _{n \rightarrow \infty} S_{n, m}=\lim _{n \rightarrow \infty}\left(\frac{1}{n+1} \sum_{k=m}^{m+n} s_{k}\right)=s
$$

uniformly with respect to $m$ (see [4]).

It can be easily verified that a convergent sequence is almost convergent and both the limits are same.

An infinite series $\sum u_{n}$ with the sequence of partial sums $\left\{s_{n}\right\}$ is said to be almost Riesz summable to $s$, if

$$
\tau_{n, m}=\frac{1}{P_{n}} \sum_{k=0}^{n} p_{k} S_{k, m} \rightarrow \text { s as } n \rightarrow \infty
$$


uniformly with respect to $m$ (see [9]).

We also use the following notations

$$
\begin{aligned}
\psi(x, t) & =\psi(t)=f(x+t)-2 f(x)+f(x-t) \\
\text { and } M_{n, m}(t) & =\frac{1}{2 \pi} \sum_{k=0}^{n} \frac{p_{k}}{(k+1) P_{n}} \frac{\sin \left((k+2 m+1) \frac{t}{2}\right) \sin \left((k+1) \frac{t}{2}\right)}{\sin ^{2}(t / 2)} .
\end{aligned}
$$

\section{Known results}

Lorentz [4] was the first who introduced the concept of almost convergence of sequences. King [3] investigated the regularity conditions for the almost summability matrices. Mazhar and Siddiqui [5] applied the concept of almost convergence of sequences to almost convergence of trigonometric sequences. In [7], Nanda introduced the spaces of strongly almost summable sequence spaces which happened to be complete paranormed spaces under certain conditions. The concept of almost convergence led to the formulation of various almost summability methods. After the definition of almost summability methods, Sharma and Qureshi [9] and Qureshi [8] determined the degree of approximation of certain functions by almost Riesz and almost Nörlund means of their Fourier series. Working in the same direction, Mishra et. al. [6] determined the degree of approximation of functions belonging to $\operatorname{Lip}(\alpha, p)$ class by almost Riesz means.

Recently, Deepmala and Piscoran [1] proved a theorem on the degree of approximation for functions belnging to $W\left(L^{p}, \xi(t)\right)(p \geq 1)$-class using almost Riesz means of its Fourier series with non-negative, non-decreasing weights $p_{n}$. They proved the following theorem:

Theorem 2.1. [1] Assume $f$ is a $2 \pi$-periodic signal (function) and integrable in the sense of Lebesgue over $[0,2 \pi]$. Then the degree of approximation of $f \in$ $W\left(L^{p}, \xi(t)\right)(p \geq 1)$-class with $0 \leq \beta \leq 1-1 / p$ by an almost Riesz means of its Fourier series is given by

$$
\left\|\tau_{n, m}(f ; x)-f(x)\right\|_{p}=O\left(P_{n}^{\beta+1 / p} \xi\left(P_{n}^{-1}\right)\right), \forall n>0,
$$

provided that the positive increasing function $\xi(t)$ has the following features:

$$
\begin{gathered}
\{\xi(t) / t\} \text { is non-increasing in } t \\
\left(\int_{0}^{\pi / P_{n}}\left(\frac{\left|\psi_{x}(t)\right|}{\xi(t)}\right)^{p} \sin ^{\beta p}(t / 2) d t\right)^{1 / p}=O(1)
\end{gathered}
$$

and

$$
\left(\int_{\pi / P_{n}}^{\pi}\left(\frac{t^{-\delta}\left|\psi_{x}(t)\right|}{\xi(t)}\right)^{p} d t\right)^{1 / p}=O\left(P_{n}^{\delta}\right)
$$

where $\delta$ is an arbitrary number such that $(\beta-\delta) q-1>0, p^{-1}+q^{-1}=1,1 \leq p \leq \infty$, and conditions (2.3) and (2.4) holds uniformly in $x$. 
Remark 2.2. We note that in the statement of Theorem 2.1, the authors have taken $p \geq 1$, but in the proof they have used the Hölder's inequality for $p>1$. Therefore, the proof is not valid for $p=1$.

Remark 2.3. For $p=\infty$, conditions (2.3) and (2.4) will not hold in the present form.

Remark 2.4. In view of the remarks of Zhang [[10], p.1140], we note that the assumption conditions $0 \leq \beta \leq 1-1 / p$ with $1 / p+1 / q=1$ and $(\beta-\delta) q-1>0$ of Theorem 2.1 imply that $\delta<0$. In this case, from condition (2.4), Theorem 2.1 is true for the function $f$ which is a constant almost everywhere and thus the result is trivial.

\section{Reformulation of the problem and main result}

Being motivated by the above remarks, we reconsider the problem of Theorem 2.1 and note that the authors defined the function class $W\left(L^{p}, \xi(t)\right)(p \geq 1)$ with the weight function $\sin ^{\beta p}(x / 2)$ whereas the deviation $\left\|\tau_{n, m}(f ; x)-f(x)\right\|_{p}$ is measured in ordinary $L_{p}$-norm. Actually, the function class $W\left(L^{p}, \xi(t)\right)$ defined in $(1.3)$ is a subclass of the weighted $L^{p}[0,2 \pi]$-space with the weight function $\sin ^{\beta p}(x / 2)$, so it is pertinent to measure the deviation in the weighted norm defined as

$$
\|f\|_{p, \beta}=\left(\frac{1}{2 \pi} \int_{0}^{2 \pi}|f(x)|^{p} \sin ^{\beta p}(x / 2) d x\right)^{1 / p}, p \geq 1 .
$$

In this paper, we reformulate the problem of Theorem 2.1 for the almost Riesz means and measure the deviation in the weighted norm defined in (3.1). More precisely, we prove

Theorem 3.1. Let $f$ be a $2 \pi$-periodic function in $W\left(L^{p}, \xi(t)\right)(p \geq 1)$-class and let $\left\{p_{n}\right\}$ be a non-negative, monotonic sequence such that

$$
(n+1) \max \left\{p_{0}, p_{n}\right\}=O\left(P_{n}\right) .
$$

Then the degree of approximation of $f$ by almost Riesz means of its Fourier series is given by

$$
\left\|\tau_{n, m}(f ; x)-f(x)\right\|_{p, \beta}=O\left(\xi\left(\frac{\pi}{n+1}\right)+(n+1)^{-\sigma}\right),
$$

where the positive increasing function $\xi(t)$ satisfies the condition

$$
t^{-\sigma} \xi(t) \text { is non-decreasing for some } 0<\sigma<1 \text {. }
$$

Note that the conditions (2.3) and (2.4) of Theorem 2.1 have been relaxed in Theorem 3.1. Also, we prove the theorem for both non-decreasing and non-increasing sequence $\left\{p_{n}\right\}$ with condition (3.2).

\section{Lemmas}

We need the following lemmas for the proof of our theorem: 
Lemma 4.1. Let $M_{n, m}(t)$ be given by (1.6). Then

$$
M_{n, m}(t)=O(n+1), \text { for } 0<t \leq \frac{\pi}{(n+1)} .
$$

Proof. For $0<t \leq \frac{\pi}{n+1}$, using $\sin (t / 2) \geq t / \pi$ and $\sin n t \leq n \sin t$, we have

$$
\begin{aligned}
\left|M_{n, m}(t)\right| & =\left|\frac{1}{2 \pi} \sum_{k=0}^{n} \frac{p_{k}}{(k+1) P_{n}} \frac{\sin \left((k+2 m+1) \frac{t}{2}\right) \sin \left((k+1) \frac{t}{2}\right)}{\sin ^{2}\left(\frac{t}{2}\right)}\right| \\
& \leq \frac{1}{2 \pi P_{n}} \sum_{k=0}^{n} \frac{p_{k}}{(k+1)} \frac{(k+1)(k+2 m+1) \sin ^{2}\left(\frac{t}{2}\right)}{\sin ^{2}\left(\frac{t}{2}\right)} \\
& =\frac{1}{2 \pi P_{n}} \sum_{k=0}^{n} p_{k}(k+2 m+1) \\
& =O(n+1) .
\end{aligned}
$$

Lemma 4.2. Let $M_{n, m}(t)$ be given by (1.6). Then

$$
M_{n, m}(t)=O\left(\frac{1}{(n+1) t^{2}}\right), \text { for } \frac{\pi}{(n+1)}<t \leq \pi .
$$

Proof. For $\pi /(n+1)<t \leq \pi$, using $\sin (t / 2) \geq t / \pi$ and $\sin n t \leq n \sin t$, we have

$$
\begin{aligned}
\left|M_{n, m}(t)\right| & =\left|\frac{1}{2 \pi} \sum_{k=0}^{n} \frac{p_{k}}{(k+1) P_{n}} \frac{\sin \left((k+2 m+1) \frac{t}{2}\right) \sin \left((k+1) \frac{t}{2}\right)}{\sin ^{2}\left(\frac{t}{2}\right)}\right| \\
& \leq \frac{1}{2 \pi P_{n}}\left|\sum_{k=0}^{n} \frac{p_{k}}{(k+1)} \frac{(k+1) \sin \left(\frac{t}{2}\right) \sin \left((k+2 m+1) \frac{t}{2}\right)}{\sin \left(\frac{t}{2}\right)} \frac{\pi}{t}\right| \\
& =\frac{1}{2 \pi P_{n} t}\left|\sum_{k=0}^{n} p_{k} \sin \left((k+2 m+1) \frac{t}{2}\right)\right| .
\end{aligned}
$$

Then, using condition (3.2), monotonicity of $\left\{p_{n}\right\}$ and Abel's lemma, we have

$$
\left|M_{n, m}(t)\right|=O\left(\frac{1}{(n+1) t^{2}}\right)
$$

in view of

$$
\left|\sum_{k=0}^{n} \sin \left((k+2 m+1) \frac{t}{2}\right)\right|=O(1 / t)
$$

Lemma 4.3. Let $g(x, t) \in L^{p}([a, b] \times[c, d]), p \geq 1$. Then,

$$
\left\{\int_{a}^{b}\left|\int_{c}^{d} g(x, t) d t\right|^{p} d x\right\}^{1 / p} \leq \int_{c}^{d}\left(\int_{a}^{b}|g(x, t)|^{p} d x\right)^{1 / p} d t
$$


This inequality is also known as the generalized form of Minkowski's inequality [[11], p.19].

\section{Proof of Theorem 3.1}

Proof. Using the integral representation of $S_{k, m}(f ; x)$ and definition of $\tau_{n, m}(f ; x)$ given in $(1.5)$, we have

$$
\begin{aligned}
\tau_{n, m}(f ; x)-f(x) & =\frac{1}{P_{n}} \sum_{k=0}^{n} p_{k}\left\{S_{k, m}(f ; x)-f(x)\right\} \\
& =\frac{1}{2 \pi P_{n}} \int_{0}^{\pi} \psi(t) \sum_{k=0}^{n} \frac{p_{k}}{(k+1)} \frac{[\cos m t-\cos (k+m+1) t]}{2 \sin ^{2}\left(\frac{t}{2}\right)} d t \\
& =\frac{1}{2 \pi P_{n}} \int_{0}^{\pi} \psi(t) \sum_{k=0}^{n} \frac{p_{k}}{(k+1)} \frac{\sin ((k+2 m+1) t / 2) \sin ((k+1) t / 2)}{\sin ^{2}\left(\frac{t}{2}\right)} d t \\
& =\int_{0}^{\pi} \psi(t) M_{n, m}(t) d t
\end{aligned}
$$

which on applying Lemma 4.3 gives

$$
\begin{aligned}
\left\|\tau_{n, m}(f ; x)-f(x)\right\|_{p, \beta} & =\left\{\frac{1}{2 \pi} \int_{0}^{2 \pi}\left|\int_{0}^{\pi} \psi(t) M_{n, m}(t) d t\right|^{p} \sin ^{\beta p}(x / 2) d x\right\}^{1 / p} \\
& \leq \int_{0}^{\pi}\left(\frac{1}{2 \pi} \int_{0}^{2 \pi}|\psi(t)|^{p} \sin ^{\beta p}(x / 2) d x\right)^{1 / p}\left|M_{n, m}(t)\right| d t
\end{aligned}
$$

Using the fact that $\psi(t) \in W\left(L^{p}, \xi(t)\right)$ due to $f \in W\left(L^{p}, \xi(t)\right)$, we have

$$
\begin{aligned}
\left\|\tau_{n, m}(f ; x)-f(x)\right\|_{p, \beta} & =\int_{0}^{\pi} O(\xi(t))\left|M_{n, m}(t)\right| d t \\
& =O(1)\left[\int_{0}^{\pi /(n+1)} \xi(t)\left|M_{n, m}(t)\right| d t+\int_{\pi /(n+1)}^{\pi} \xi(t)\left|M_{n, m}(t)\right| d t\right] \\
& =I_{1}+I_{2}(\text { say }) .
\end{aligned}
$$

Using Lemma 4.1, increasing nature of $\xi(t)$ and the mean value theorem for integrals, we have

$$
\begin{aligned}
I_{1} & =O(1) \int_{0}^{\pi /(n+1)} \xi(t)\left|M_{n, m}(t)\right| d t=O(1) \int_{0}^{\pi /(n+1)}(n+1) \xi(t) d t \\
& =O\left(\xi\left(\frac{\pi}{n+1}\right)\right) .
\end{aligned}
$$


Using Lemma 4.2, condition (3.4) and the mean value theorem for integrals, we have

$$
\begin{aligned}
I_{2} & =O(1) \int_{\pi /(n+1)}^{\pi} \xi(t)\left|M_{n, m}(t)\right| d t \\
& =O(1) \frac{1}{(n+1)} \int_{\pi /(n+1)}^{\pi} \frac{t^{\sigma}}{t^{2}} \frac{\xi(t)}{t^{\sigma}} d t=O(1) \frac{\xi(\pi) \pi^{-\sigma}}{(n+1)}\left(\frac{\pi}{n+1}\right)^{\sigma-1} \\
& =O\left((n+1)^{-\sigma}\right) .
\end{aligned}
$$

Collecting (5.1) - (5.3), we have

$$
\left\|\tau_{n, m}(f ; x)-f(x)\right\|_{p, \beta}=O\left(\xi\left(\frac{\pi}{n+1}\right)+(n+1)^{-\sigma}\right) .
$$

\section{Corollaries}

For $\beta=0$, the weighted class $W\left(L^{p}, \xi(t)\right)$ reduces to $\operatorname{Lip}(\xi(t), p)$. Thus, we have the following corollary:

Corollary 6.1. Let $f$ be a $2 \pi$-periodic function in $\operatorname{Lip}(\xi(t), p)(p \geq 1)$-class and let $\left\{p_{n}\right\}$ be a non-negative, monotonic sequence such that

$$
(n+1) \max \left\{p_{0}, p_{n}\right\}=O\left(P_{n}\right) .
$$

Then the degree of approximation of $f$ by almost Riesz means of its Fourier series is given by

$$
\left\|\tau_{n, m}(f ; x)-f(x)\right\|_{p}=O\left(\xi\left(\frac{\pi}{n+1}\right)+(n+1)^{-\sigma}\right),
$$

where the positive increasing function $\xi(t)$ satisfies the condition

$$
t^{-\sigma} \xi(t) \text { is non-decreasing for some } 0<\sigma<1 \text {. }
$$

If $\beta=0$ and $\xi(t)=t^{\alpha}$, then the weighted class $W\left(L^{p}, \xi(t)\right)(p \geq 1)$ reduces to the class $\operatorname{Lip}(\alpha, p)(p \geq 1)$. In this case, the function $t^{-\sigma} \xi(t)=t^{\alpha-\sigma}$ is increasing for $0<\sigma<\alpha \leq 1$. Thus, we have the following corollary:

Corollary 6.2. Let $f$ be a $2 \pi$-periodic function in $\operatorname{Lip}(\alpha, p)(p \geq 1)$-class and let $\left\{p_{n}\right\}$ be a non-negative, monotonic sequence such that

$$
(n+1) \max \left\{p_{0}, p_{n}\right\}=O\left(P_{n}\right) .
$$

Then the degree of approximation of $f$ by almost Riesz means of its Fourier series is given by

$$
\left\|\tau_{n, m}(f ; x)-f(x)\right\|_{p}=O\left((n+1)^{-\sigma}\right), 0<\sigma<\alpha .
$$

However, we can obtain the degree of approximation of a function $f \in \operatorname{Lip}(\alpha, p)$ independently as under:

Putting $\xi(t)=t^{\alpha}$ in (5.1), we have

$$
I_{1}=O(1) \int_{0}^{\pi /(n+1)}(n+1) t^{\alpha} d t=O\left((n+1)^{-\alpha}\right), 0<\alpha \leq 1
$$


and

$$
I_{2}=O(1 /(n+1)) \int_{\pi /(n+1)}^{\pi} \frac{t^{\alpha}}{t^{2}} d t= \begin{cases}O\left((n+1)^{-\alpha}\right), & 0<\alpha<1 \\ O\left(\frac{\log (n+1)}{n+1}\right), & \alpha=1\end{cases}
$$

Combining (6.1) and (6.2), we have

$$
\left\|\tau_{n, m}(f ; x)-f(t)\right\|_{p}= \begin{cases}O\left((n+1)^{-\alpha}\right), & 0<\alpha<1 \\ O\left(\frac{\log (n+1)}{n+1}\right), & \alpha=1\end{cases}
$$

If $\beta=0, \xi(t)=t^{\alpha}$ and $p \rightarrow \infty$, then the weighted class $W\left(L^{p}, \xi(t)\right)(p \geq 1)$ reduces to the class $\operatorname{Lip}(\alpha)$. Thus, we have the following corollary:

Corollary 6.3. Let $f$ be a $2 \pi$-periodic function in Lip $(\alpha)$ class and let $\left\{p_{n}\right\}$ be a nonnegative, monotonic sequence such that

$$
(n+1) \max \left\{p_{0}, p_{n}\right\}=O\left(P_{n}\right) .
$$

Then the degree of approximation of $f$ by almost Riesz means of its Fourier series is given by

$$
\left\|\tau_{n, m}(f ; x)-f(x)\right\|_{\infty}=O\left((n+1)^{-\sigma}\right), 0<\sigma<\alpha .
$$

Independently, we can obtain

$$
\left\|\tau_{n, m}(f ; x)-f(x)\right\|_{\infty}= \begin{cases}O\left((n+1)^{-\alpha}\right), & 0<\alpha<1 \\ O\left(\frac{\log (n+1)}{n+1}\right), & \alpha=1\end{cases}
$$

\section{References}

[1] Deepmala, Piscoran, L.I., Approximation of signals (functions) belonging to certain Lipschitz classes by almost Riesz means of its Fourier series, J. Inequal. Appl., (2016), 2016:163.

[2] Khan, H.H., A note on a theorem of Izumi, Commun. Fac. Sci. Math, Ankara, Turkey, 31(1982), 123-127.

[3] King, J.P., Almost summable sequences, Proc. Amer. Math. Soc., 17(1966), 1219-1225.

[4] Lorentz, G.G., A contribution to the theory of divergent series, Acta Math., 80(1948), 167-190.

[5] Mazhar, S.M., Siddiqui, A.H., On almost summability of a trigonometric sequence, Acta Math. Hungar., 20(1969), no. 1-2, 21-24.

[6] Mishra, V.N., Khan, H.H., Khan, I.A., Mishra, L.N., On the degree of approximation of signals Lip $(\alpha, p),(p \geq 1)$ class by almost Riesz means of its Fourier series, J. Class. Anal., 4(2014), no. 1, 79-87.

[7] Nanda, S., Some sequence space and almost convergence, J. Austral. Math. Soc., A 22(1976), 446-455.

[8] Qureshi, K., On the degree of approximation of a periodic function $f$ by almost Nörlund means, Tamkang J. Math., 12(1981), no. 1, 35-38.

[9] Sharma, P.L., Qureshi, K., On the degree of approximation of a periodic function by almost Riesz means, Ranchi Univ. Math., 11(1980), 29-43.

[10] Zhang, R.J., On the trigonometric approximation of the generalized weighted Lipschitz class, Appl. Math. Comput., 247(2014), 1139-1140. 
[11] Zygmund, A., Trigonometric Series, Cambridge University Press, Cambridge, 2002.

Uaday Singh

Department of Mathematics

Indian Institute of Technology Roorkee

Roorkee-247667, India

e-mail: uadayfma@iitr.ac.in

Arti Rathore

Department of Mathematics

Indian Institute of Technology Roorkee

Roorkee-247667, India

e-mail: artirathore201@gmail.com 